\title{
A Study on Risk Management of E-commerce Ecological Chain
}

\author{
Chengcheng Zhao* \\ School of Management and Engineering, Zheng Zhou University, No.100 Science Avenue,Zhengzhou , \\ Henan, China \\ Chengcheng1103999@163.com
}

Keywords: Ecological Chain; Ecological Theory; Node

\begin{abstract}
With the "the Belt and Road Initiative", economic development zone's construction and technological innovation, e-commerce also showed a rapid development trend. The unprecedented opportunities for the electricity industry have also brought challenges. The development of the eco-chain value chain, the supply chain and the industrial chain have also gradually presented some drawbacks. Therefore, this paper aims at the risk of ecological chain of e-commerce, the risk identification and prevention, and promotes the healthy development of electric business enterprises.
\end{abstract}

\section{INTRODUCTION}

The popularity of the Internet and the improvement of the level of modern logistics, to promote Chinese ecommerce ecosystem continued to move forward. With the "One Belt and One Road Initiative" economic development zone construction, countries focus on the establishment of Chinese economic and trade relations, cross-border electricity industry to gain an unprecedented opportunity. October 2017, in China, the first of "One Belt and One Road Initiative" noneconomic and trade cooperation B2B business platform will be settled in Wuhou District. At the same time, it is reported that Chinese first cross-border electric power factory in Hangzhou, the first half of this year to achieve cross-border e-commerce transactions amounted to 4.457 billion dollars, Hangzhou has become an important driving force for steady growth. According to the China Electronic Commerce Research Center monitoring data show that in the first half of 2017 Chinese cross-border imports of electricity business size reached 862.4 billion RMB (including imports of B2B and import B2C), an increase of $66.3 \%$, is expected in the end of 2017 will reach 185.43 billion RMB. However, the electricity business enterprises are also facing a serious crisis. August 23rd, PayPal joint research and consulting firm Dataway released a report shows that $49 \%$ of Chinese consumers choose to overseas direct purchase in the form of sea Amoy, but the prominent fake problem led to consumer confidence crisis. From the Jindong Gucci change Gucc event, to the tiger Tiger Jindong global purchase fake to foreign wharf "400 RMB fake to sell 7,000 RMB nobody, buyers rely on loopholes can be make 100,000 RMB per month," the report, in successive of the sea Amoy fake incident exposure, the electricity industry suffered a credit crisis. The crisis of trust is only a risk of the development of electricity business ecosystem, if we want to develop steadily, we must establish a complete risk system. The industry vertical and horizontal integration, coordinated development.

At present, for the research of e-commerce ecosystem, a certain theoretical research framework has been formed at home and abroad. The contents and research hotspots mainly include the connotation and characteristics of e-commerce information ecosystem, ecological factors and interrelations, ecological environment, e-commerce Ecological system construction and management issues. Foreign scholars Javalgi RG, Todd PR and Scherer RF proposed and mapped an e-commerce eco-model based on the current global e-commerce environment using the organizational ecology dynamics of the classical model in the Internet ecosystem(Javalgi et al, 2005), Assadourian E through the world economy Environmental analysis, the development of e-commerce can't be separated from the stability of the economic environment to support the country's economic environment is one of the conditions that constitute the ecological environment of ecommerce (Assadourian,2008), Chinese scholar Min Xilin and Yao Rui on e-commerce ecosystem in the middle Businessmen, traders and managers, and put forward the development strategy to be taken in promoting the development of e-commerce ecosystem (Xilin et al., 2007). Based on the theory of E-commerce information ecosystem, this paper further analyses the three subsystems - e-commerce information field, e-commerce information ecology chain and e-commerce information ecosystem, and then builds e-commerce information ecosystem (Keyan Yang, 2014). Foreign scholars focus on e-commerce risk research focused on e-commerce security. Warren M proposed that e-commerce security is a complex issue, by improving the level of ecommerce technology and organizational level, can reduce the enterprise e-commerce security issues (Warren M,1993). There are few literatures on the risk of e-commerce ecology chain from the information ecosystem, mainly focusing on the risk management research and the risk management research of ecommerce ecosystem in the e-commerce environment. Yang Xiaomei and other analysis of the e-commerce environment e-commerce platform for the credit risk, through the risk management research C2C e-commerce site (Xiaomei et al., 2009). Liu Xueli analyses the risk factors of E-commerce ecosystem, and puts forward the relevant methods to evaluate the risk of e-commerce 
ecosystem, and the fuzzy comprehensive evaluation method can deal with the ambiguity of e-commerce ecosystem risk (Xueli Liu, 2011).

From the current research situation, the theoretical research level from the perspective of information ecology chain of e-commerce risk prevention research results are relatively small. Therefore, the research of this paper aims at the theoretical level to expand the application of information ecology theory in the field of electronic commerce, so as to help enterprises to carry out e-commerce to improve its core competitiveness.

\section{RISK MANAGEMENT OF E-COMMERCE ECOLOGY CHAIN}

Under the contemporary society, the life of the public has been closely linked with the Internet, a large number of individuals, governments, enterprises are involved in e-commerce, research e-commerce activities of great significance. The relationship between e-commerce is not a single existence and development, and the interdependency relationship between e-commerce forms a chain model. It is effective to improve the survivability and evolution of e-commerce subject according to the ecology theory. space. The uncertainty of risk and the link between risk and corporate interests determine the need for e-commerce to study the risks in e-commerce. The risk of e-commerce ecological chain contains the risk of e-commerce environment to a certain extent. Therefore, it is very important to study the risk of ecommerce ecology chain according to the relevant knowledge of risk management in management theory.

E-commerce eco-chain is a set of e-commerce value chain, e-commerce products supply chain, e-commerce industry chain of a special network information ecological chain. In e-commerce, e-commerce business of each e-commerce activities can create value, these interrelated e-commerce activities will constitute the creation of e-commerce value of a dynamic process, that is, e-commerce value chain. If the e-commerce value chain reflects the value-added process of e-commerce products and services, then the e-commerce supply chain is a product chain around the end product and connecting e-commerce providers and e-commerce products consumers. Industrial chain is the same industry or different industries of enterprises, to the product as the object, to input and output as a link to the value of valueoriented to meet user needs as the goal, based on specific logical and spatial and temporal layout of the formation of the upper and lower associated, Dynamic chain organization. The chains formed by e-commerce are linked by multiple nodes, and the nodes and nodes are interdependent. The interaction between nodes determines the structural relationship of e-commerce ecological chain.

\subsection{E-commerce ecological chain risk analysis}

The risk identification of e-commerce ecology chain is to find out the risk of e-commerce ecological chain formation, study the risk of circulation efficiency in the process of e-commerce ecological chain, and then explore the internal organization form and the external display effect risk. At present, the risk identification of e-commerce ecological chain mainly includes four kinds of risk forms: structural fracture, running paralysis, organizational aging and negative image.

Structural fracture. E-commerce ecological chain structure Rupture risk refers to the interruption of the chain-dependent relationship formed by the main body of electronic commerce. E-commerce ecological chain structure of the risk of fracture can be expressed in the chain of nodes does not exist or the chain of nodes in the relationship between the rupture. E-commerce ecological chain is an organic link whole. The main body through the commodity flow, information flow, capital flow and logistics flow to produce contact. The normal flow between them is a necessary condition to ensure the normal operation of e-commerce ecological chain. The core node to withdraw or not be able to participate in the operation of the entire ecological chain, it will cause the lack of commodity flow, capital flow and information flow break, and then lead to risk.

Running paralyzed. E-commerce ecological chain paralysis risk manifestations of the general network information system paralysis, logistics and distribution paralysis and capital chain paralysis. Any part of the paralysis will inevitably lead to the entire ecological chain stagnation, and even may lead to the collapse of ecommerce ecosystem.

Organization aging. E-commerce ecological chain aging performance in the e-commerce ecological chain did not break, but also in the normal operation, but the chain appears in the flow rate decline or some of the concept of backward function and so on. These phenomena will lead to e-commerce ecological chain of logistics organizations, information organizations, financial organization aging and node structure of the aging.

Negative image. The image of e-commerce ecological chain is a kind of intuitive expression in the social and economic environment. The poor image mainly refers to the low reputation of e-commerce ecological chain, low reputation and large image deviation. These low-level nodes are not recognized by the public, resulting in the chain of information transmission efficiency is low, lack of enthusiasm; low reputation of the e-commerce ecological chain will cause the chain node instability, some nodes in order to pursue their own interests to the reputation Good chain; the image is large because the chain of their own image will not manage the third party will make the impression of bias, so even if the chain of negative image is small but the public is caught in the circle of thinking do not want to accept it.

\subsection{Risk management prevention and governance}

E-commerce ecological chain risk management measures is the main body of e-commerce through a series of means and strategies to prevent and reduce risk. The specific goal is to prevent risks as much as possible, while taking effective strategies for unavoidable risks to minimize the impact of risk. In this paper, from the prevention and management of two aspects of risk management, risk prevention is a risk awareness training and the establishment of risk behaviour guidelines, 
governance risk is to reduce the risk of risk, transfer risk loss and avoid the risk of uncontrollable The

Enhance risk awareness. E-commerce ecological chain awareness refers to the spontaneous risk of each node to generate predictive thinking and behaviour. And then expand the awareness of risk prevention to the entire ecological chain of the various nodes. Drag the local to the overall risk awareness of the expansion, and gradually establish a culture of risk prevention. Each node in the ecological chain can be classified according to the main body position, carry out risk group discussion, popularize risk prevention knowledge. At the same time can carry out risk simulation rehearsal, the risk prevention awareness within the organization.

Establish a risk early warning system. The risk early warning system can be compared with the existing risk database, monitor the trend of the risk factors, analyse the risk state and the development drive, and estimate the risk based on the risk assessment index can't happen. A sound risk early warning system should have a complete risk public opinion database, and the risk management organization has a long-term and organized collection of risk history and research status. A comprehensive risk early warning system can increase the accuracy of the early warning system, estimate the probability of occurrence of risk, identify the risk of early warning coordinates and predict the severity of the risk.

Improve risk prevention measures. Optimize the combination of nodes, and consolidate the dependencies between nodes. Reasonable selection of nodes and training nodes, adjust the node combination makes the node distribution appropriate. Establish incentive mechanism, encourage each node to take the initiative to carry out risk prevention, effectively curb the risk of occurrence of passive. Update technology and equipment, the use of new technologies, new equipment, improve operational efficiency, to ensure smooth operation, and thus attract more capital investment. Maintain their own image, good image management of the node, more likely to attract capital, technology, talent to enter, is conducive to strengthening the stability of the ecological chain, to promote the healthy and healthy development of ecological chain.

\section{CONCLUSIONS}

Based on the theory of ecology and risk management, this paper analyses the risk of e-commerce ecological chain, summarizes the risks existing in the structure, circulation, internal organization and external image of e-commerce ecological chain, and puts forward the corresponding risk prevention and governance basic strategy. Then, using this to improve the ecological chain construction, expand the connotation of e-commerce information ecological chain. This paper has enriched the theoretical research of e-commerce ecological chain, and it is of great significance to promote the research of e-commerce ecology chain risk management. It is of great significance to promote the information balance and common development of e-commerce information subject and improve the coordinated development of ecommerce ecosystem significance.

\section{REFERENCES}

[1] Assadourian E,2008. Global economic growth continues at expense of ecological systems, WorldWatch. 21(3), pp.30-31. [2] Javalgi R G, Todd P R, Scherer R F, 2005. The dynamics of global ecommerce an organizational ecology perspective, International Marketing Review. 22(4), pp.420-435.

[3] Keyan Yang, 2014. Research on Information Ecosystem Construction about E-business, Information Science. (3), pp.37-41.

[4] Warren M, 1993. A security risk management approach for e-commerce, Information Management\&Computer Security. 11(5), pp.238-242.

[5] Xiaomei Yang, Jiye Liang, Hong Jia. Research on Credit Risk Management Strategy Based on CtoC E-commerce Website in China[J]. Library and Information Service, 2009(8):126-129.

[6] Xilin Min, Rui Yao, 2007. Analysis on the Role Development of the Subject in E-commerce Ecosystem, .EBusiness Journal. (6), pp.115-116.

[7] Xueli Liu, 2011. Research on Risk Assessment of Ecommerce Ecosystem, Journal of Commercial Economics. (30), pp.43-44. 